\title{
SOLUTION OF ONE INITIAL-BOUNDARY WENTZEL PROBLEM FOR A PARABOLIC EQUATION WITH DISCONTINUOUS COEFFICIENTS BY THE BOUNDARY INTEGRAL EQUATION METHOD
}

\author{
Bohdan Kopytko ${ }^{1}$, Zhanneta Tsapovska ${ }^{2}$ \\ ${ }^{I}$ Institute of Mathematics, Czestochowa University of Technology \\ Czestochowa, Poland \\ ${ }^{2}$ Ivan Franko Lviv National University \\ Lviv, Ukraine \\ bohdan.kopytko@gmail.com,tzhannet@yahoo.com
}

Received: 11 January 2017; accepted: 10 March 2017

\begin{abstract}
In this article we consider the question of existence in the Holder class of the solution of the initial-boundary problem for a linear parabolic second-degree equation with discontinuous coefficients in noncylindrical domain. This domain is bounded of the smooth elementary surfaces of the Holder class $\mathrm{H}^{2+\lambda,(2+\lambda) / 2}$. The boundary conditions and the conjugation condition of the Wentzel type are given to external and internal boundaries of a domain respectively. We use the potential method to solve this problem.
\end{abstract}

MSC 2010: $35 K 20$

Keywords: linear parabolic second-degree operator, potential method, conjugation condition of Wentzel type

\section{Introduction}

The theory of potentials is very important in the study of the Cauchy problem, the boundary-value problem, the conjugation problems for the heat equations and the general second-degree parabolic equations as well. The potential method is used to thoroughly examine the initial-boundary problems for the uniformly parabolic equations when the order of the differential boundary operators is less than the order of the equation in the domain [1-8]. We can encounter the initialboundary problems which contain derivatives of the second and higher orders. The Wentzel problem is a vivid example of this type [9]. This is the initialboundary problem for the parabolic equation with the boundary condition which has the form of a parabolic operator on the tangential variables. That problem arises, in particular, into the theory of Markov processes in the construction of a diffusion process in a domain on predetermined the diffusion coefficients and the boundary conditions. 
The parabolic initial-boundary Wentzel problem (in a cylindrical domain) was investigated in the works [10-12] by the methods of functional analysis. In the papers $[13,14]$ (in cylindrical and noncylindrical domains) this problem was studied by the boundary integral equation method using a simple-layer potential. As for the parabolic problem with Wentzel conjugation conditions, this problem, for the case of a cylindrical domain, is studied in the most general formulation in the papers $[15,16]$.

In this article, we consider one of the problems in the assumption that the boundaries of the domains are the elementary noncylindrical surfaces of the Holder class.

\section{Problem statement and its solution}

In a layer $R_{T}^{n+1}=R^{n} \times(0, T)$, where $T>0$ is fixed, $R^{n}, n \geq 2$, is the $n$-dimensional Euclidian space of the points $x=\left(x^{\prime}, x_{n}\right), x^{\prime}=\left(x_{1}, \ldots, x_{n-1}\right) \in R^{n-1}$, we consider the domain $\Omega=\left\{(x, t) \in R_{T}^{n+1} \mid F_{1}\left(x^{\prime}, t\right)<x_{n}<F_{2}\left(x^{\prime}, t\right)\right\}$ with the smooth boundary $\partial \Omega=\Sigma_{1} \cup \Sigma_{2}$, where $\Sigma_{m}=\left\{(x, t) \in R_{T}^{n+1} \mid x_{n}=F_{m}\left(x^{\prime}, t\right)\right\}, \quad m=1,2$. We assume, that the surface $\Sigma_{0}=\left\{(x, t) \in R_{T}^{n+1} \mid x_{n}=F_{0}\left(x^{\prime}, t\right)\right\}$ subdivides the domain $\Omega$ into two domains $\Omega_{1}$ and $\Omega_{2}$ with the boundaries $\partial \Omega_{m}=\Sigma_{0} \cup \Sigma_{m}, m=1,2$, and $F_{1}\left(x^{\prime}, t\right)<F_{0}\left(x^{\prime}, t\right)<F_{2}\left(x^{\prime}, t\right)$.

Let $\quad D_{m}=\Omega_{m} \cap\{t=0\}, m=1,2, S_{\tau}^{(m)}=\Sigma_{m} \cap\{t=\tau\}, \tau \in[0, T], m=0,1,2$, in particular by $S_{m}, \quad m=0,1,2$, we denote $S_{\tau}^{(m)}$ at $\tau=0$. By $\mathbf{v}^{(m)}(y, \tau)=\left(v_{1}^{(m)}(y, \tau), \ldots, v_{n}^{(m)}(y, \tau)\right)$ we denote the unit normal vector at the point $(y, \tau)$ to the surface $S_{\tau}^{(m)}, m=0,1,2$, which is in the section $t=\tau$, and the vectors $\mathbf{v}^{(1)}(y, \tau)$ and $\mathbf{v}^{(0)}(y, \tau), \mathbf{v}^{(2)}(y, \tau)$ directed inwards to the domains $\Omega_{1}$ and $\Omega_{2}$ respectively. $\bar{v}\left(x^{\prime}, t\right)$ is the value of the function $v(x, t)$ on the surface $\Sigma_{m}$, $m=0,1,2$, i.e. $\bar{v}(x, t) \equiv v\left(x^{\prime}, F_{m}\left(x^{\prime}, t\right), t\right)$.

The differential operators with respect to $t$ and $x_{i}$ we denote by $D_{t}$ and $D_{i}$, $\nabla=\left(D_{1}, \ldots, D_{n}\right), \delta_{i}^{(m)}(i=1, \ldots, n)$ is a tangent differential operator on $\Sigma_{m}$, $m=0,1,2$, i.e. $\delta_{i}^{(m)}=\sum_{k=1}^{n} \tau_{i k}^{(m)} D_{k}$, where $\tau_{i k}^{(m)}=\delta_{i}^{k}-v_{i}^{(m)} v_{k}^{(m)}, i, k=1, \ldots, n, \delta_{i}^{k}$ is the Kronecker symbol. If $x \in R^{n}, y \in R^{n}$, then $(x, y)=\sum_{i=1}^{n} x_{i} y_{i}$.

Let $B \subset R^{n}$ and $Q \subset R_{T}^{n+1}$ be any domains, $\bar{B}$ and $\bar{Q}$ are the closure of them, $l$ and $\lambda$ are some numbers, $l \geq 0$ is an integer and $0<\lambda<1$. 
Similar to [3, ch. I, § 1] $\mathrm{H}^{l+\lambda,(l+\lambda) / 2}$ is the class surface, $\mathrm{H}^{l+\lambda}(\bar{B})$ and $\mathrm{H}^{l+\lambda,(l+\lambda) / 2}(\bar{Q})$ are the corresponding Holder spaces with the norms $\|\varphi\|_{\mathrm{H}^{l+\lambda}(\bar{B})}$ and $\|\psi\|_{\mathrm{H}^{l+\lambda,(l+\lambda) / 2}(\bar{Q})}$ which are defined on $\bar{B}$ and $\bar{Q}$ respectively. $\mathrm{H}_{0}^{l+\lambda,(l+\lambda) / 2}(\bar{Q})$ is the subspace of functions from $\mathrm{H}^{l+\lambda,(l+\lambda) / 2}(\bar{Q})$ that together with admissible derivative with respect to the time variable, vanishes at $t=0 . C, c$ are positive constants independent of $(x, t)$. We are not interested in their specific value.

In a layer $R_{T}^{n+1}$, let us consider two second-order uniformly parabolic equations

$$
\begin{array}{r}
L_{m} u_{m} \equiv \sum_{i, j=1}^{n} a_{i j}^{(m)}(x, t) D_{i} D_{j} u_{m}+\sum_{i=1}^{n} a_{i}^{(m)}(x, t) D_{i} u_{m}+a_{0}^{(m)}(x, t) u_{m}-D_{t} u_{m}=0, \\
m=1,2 .
\end{array}
$$

Assume that the coefficients of the operator $L_{m}, m=1,2$, are defined in $\bar{R}_{T}^{n+1}$ and the following assumptions are true:

(A1) $\sum_{i, j=1}^{n} a_{i j}^{(m)}(x, t) \xi_{i} \xi_{j} \geq \delta_{0 m}|\xi|^{2}, a_{i j}^{(m)}=a_{j i}^{(m)}, \delta_{0 m}>0, m=1,2, \forall(x, t) \in \bar{R}_{T}^{n+1}$, $\forall \xi \in R^{n}$;

(A2) $a_{i j}^{(m)}, a_{i}^{(m)}, a_{0}^{(m)} \in H^{\lambda, \lambda / 2}\left(\bar{R}_{T}^{n+1}\right), m=1,2, i, j=1, \ldots, n$.

Assumptions (A1), (A2) guarantee the existence of a fundamental solution (f.s.) for each equation from (1) (see [3, ch. IV, § 11]) which we will denote by $G^{(m)}(x, t ; \xi, \tau),\left(x, \xi \in R^{n}, 0 \leq \tau<t \leq T\right), m=1,2$.

Let us consider the integrals - the parabolic simple-layer potentials:

$$
\begin{gathered}
u_{m}^{(0)}(x, t)=\int_{0}^{t} d \tau \int_{S_{\tau}^{(0)}} G^{(m)}(x, t ; \xi, \tau) V_{m}(\xi, \tau) d s_{\xi}, \quad(x, t) \in \bar{R}_{T}^{n+1}, \quad m=1,2, \\
u_{m}^{(1)}(x, t)=\int_{0}^{t} d \tau \int_{S_{\tau}^{(m)}} G^{(m)}(x, t ; \xi, \tau) V_{m+2}(\xi, \tau) d s_{\xi}, \quad(x, t) \in \bar{R}_{T}^{n+1}, \quad m=1,2,
\end{gathered}
$$

where the functions $V_{m}$ and $V_{m+2}, m=1,2$, defined, bounded and continuous on surfaces $\Sigma_{0}$ and $\Sigma_{m}$ respectively.

We note some properties of the potential (2), (3) (see [1-4]). The functions (2) and (3) satisfy the equation (1) at each point $(x, t) \in R_{T}^{n+1} \backslash \Sigma_{0}$ and $(x, t) \in R_{T}^{n+1} \backslash \Sigma_{m}$ respectively, and they also satisfy the initial condition $u_{m}^{(j)}(x, 0)=0, x \in R^{n}$, $j=0,1 ; m=1,2$. 
At the points of the surfaces $\Sigma_{0}$ and $\Sigma_{m}, m=1,2$, let the conormal vectors $\mathbf{N}^{(0 m)}(x, t)=\mathbf{A}^{(m)}(x, t) \mathbf{v}^{(0)}(x, t), \quad(x, t) \in \Sigma_{0}, \quad$ and $\quad \mathbf{N}^{(m)}(x, t)=\mathbf{A}^{(m)}(x, t) \mathbf{v}^{(m)}(x, t)$, $(x, t) \in \Sigma_{m}$, where $\mathbf{A}^{(m)}(x, t)=\left(a_{i j}^{(m)}(x, t)\right)_{i, j=1}^{n}, m=1,2$, be defined. The important property of the simple-layer potential reflected in the boundary relations for the conormal derivative of this potential (see [2, ch. V, § 2], [3, ch. IV, § 15], [4, 5]).

Using a f.s. $G^{(m)}(x, t ; \xi, \tau)$ we can identify and explore in the unbounded domain the properties of two integrals connected with the operator $L_{m}, m=1,2$ :

$$
\begin{gathered}
u_{m}^{(2)}(x, t)=\int_{0}^{t} d \tau \int_{R^{n}} G^{(m)}(x, t ; \xi, \tau) f_{m}(\xi, \tau) d \xi, \quad m=1,2, \\
u_{m}^{(3)}(x, t)=\int_{R^{n}} G^{(m)}(x, t ; \xi, 0) \varphi_{m}(\xi) d \xi, \quad m=1,2 .
\end{gathered}
$$

The first integral is called the volumetric potential and the second one is called the Poisson potential. If specified functions $f_{m}(x, \tau), x \in R^{n}, \tau \in[0 ; T]$ and $\varphi_{m}(x)$, $x \in R^{n}, m=1,2$, are bounded and continuous, $f_{m}(x, \tau)$ satisfies the Holder condition for variable $x$ uniformly relative to $\tau \in[0 ; T]$ then the function $u_{m}^{(2)}(x, t)$ satisfies the equation

$$
L_{m} u_{m}^{(2)}(x, t)=-f_{m}(x, t), \quad m=1,2,
$$

in the domain $(x, t) \in R_{T}^{n+1}$ with a zero initial condition $u_{m}^{(2)}(x, 0)=0, x \in R^{n}$, and the function $u_{m}^{(3)}(x, t)$ satisfies equation (1) in the same domain with the initial condition

$$
u_{m}^{(3)}(x, 0)=\varphi_{m}(x), \quad x \in R^{n}, \quad m=1,2 .
$$

Considering this, we can give the general classical solution of the Cauchy problem (6), (7) as the sum of potentials (4), (5). And if $f_{m} \in \mathrm{H}^{\lambda, \lambda / 2}\left(\bar{R}_{T}^{n+1}\right)$, $\varphi_{m} \in \mathrm{H}^{2+\lambda}\left(R^{n}\right)$, then the potentials (4), (5) and therefore the solution of the problem (6), (7) belongs to the Holder class $\mathrm{H}^{2+\lambda,(2+\lambda) / 2}\left(\bar{R}_{T}^{n+1}\right)$.

We will consider the following conjugation problem: we have to find the function $u(x, t)=u_{m}(x, t),(x, t) \in \bar{\Omega}_{m}, m=1,2$, based on the conditions

$$
\begin{gathered}
L_{m} u_{m}(x, t)=-f_{m}(x, t), \quad(x, t) \in \Omega_{m}, \quad m=1,2, \\
u_{m}(x, 0)=\varphi_{m}(x), \quad x \in D_{m}, \quad m=1,2, \\
L_{3} u(x, t) \equiv u_{1}(x, t)-u_{2}(x, t)=z(x, t), \quad(x, t) \in \Sigma_{0} \backslash S_{0},
\end{gathered}
$$




$$
\begin{gathered}
L_{4} u(x, t) \equiv \sum_{i, j=1}^{n} \beta_{i j}^{(0)}(x, t) \delta_{i}^{(0)} \delta_{j}^{(0)} u_{1}-\left(\boldsymbol{\beta}^{(01)}(x, t), \nabla u_{1}\right)+\beta_{0}^{(01)}(x, t) u_{1}-D_{t} u_{1}+ \\
+\left(\boldsymbol{\beta}^{(02)}(x, t), \nabla u_{2}\right)+\beta_{0}^{(02)}(x, t) u_{2}=\theta(x, t), \quad(x, t) \in \Sigma_{0} \backslash S_{0}, \\
L_{5} u_{1}(x, t) \equiv \sum_{i, j=1}^{n} \beta_{i j}^{(1)}(x, t) \delta_{i}^{(1)} \delta_{j}^{(1)} u_{1}+\left(\boldsymbol{\beta}^{(1)}(x, t), \nabla u_{1}\right)+\beta_{0}^{(1)}(x, t) u_{1}- \\
-D_{t} u_{1}=\psi_{1}(x, t), \quad(x, t) \in \Sigma_{1} \backslash S_{1}, \\
L_{6} u_{2}(x, t) \equiv \sum_{i, j=1}^{n} \beta_{i j}^{(2)}(x, t) \delta_{i}^{(2)} \delta_{j}^{(2)} u_{2}+\left(\boldsymbol{\beta}^{(2)}(x, t), \nabla u_{2}\right)+\beta_{0}^{(2)}(x, t) u_{2}- \\
-D_{t} u_{2}=\psi_{2}(x, t), \quad(x, t) \in \Sigma_{2} \backslash S_{2},
\end{gathered}
$$

where $\boldsymbol{\beta}^{(0 m)}(x, t)=\left(\beta_{i}^{(0 m)}(x, t)\right)_{i=1}^{n}, \boldsymbol{\beta}^{(m)}(x, t)=\left(\beta_{i}^{(m)}(x, t)\right)_{i=1}^{n}, m=1,2$.

We assume that for the coefficients of the Wentzel type operators $L_{4}, L_{5}$ and $L_{6}$ the following conditions hold:

(B1) $\sum_{i, j=1}^{n} \beta_{i j}^{(m)}(x, t) \xi_{i} \xi_{j} \geq \mu_{0 m}|\xi|^{2}, \quad \beta_{i j}^{(m)}(x, t)=\beta_{j i}^{(m)}(x, t), \quad \mu_{0 m}>0, \quad \forall(x, t) \in \Sigma_{m}$, $\forall \xi \in R^{n}, \quad \xi \perp \mathbf{v}^{(m)}(x), \quad m=0,1,2 ;$

(B2) $\beta_{i j}^{(0)}, \beta_{i}^{(0 m)}, \beta_{0}^{(0 m)} \in \mathrm{H}^{\lambda, \lambda / 2}\left(\Sigma_{0}\right), i, j=1, \ldots, n, \quad\left(\boldsymbol{\beta}^{(0 m)}, \mathbf{v}^{(0)}\right) \geq 0, \quad m=1,2$; $\beta_{i j}^{(m)}, \beta_{i}^{(m)}, \beta_{0}^{(m)} \in \mathrm{H}^{\lambda, \lambda / 2}\left(\Sigma_{m}\right), i, j=1, \ldots, n,\left(\boldsymbol{\beta}^{(m)}, \mathbf{v}^{(m)}\right) \geq 0, \quad m=1,2$.

Also we assume, that

$$
\begin{gathered}
\Sigma_{0}, \Sigma_{m} \in \mathrm{H}^{2+\lambda,(2+\lambda) / 2}, \quad \rho\left(\Sigma_{0}, \Sigma_{m}\right) \geq d_{0 m}>0, \quad m=1,2, \\
f_{m} \in \mathrm{H}^{\lambda, \lambda / 2}\left(\bar{R}_{T}^{n+1}\right), \quad \varphi_{m} \in \mathrm{H}^{2+\lambda}\left(R^{n}\right), \quad m=1,2, \\
z \in \mathrm{H}^{2+\lambda,(2+\lambda) / 2}\left(\Sigma_{0}\right), \quad \theta \in \mathrm{H}^{\lambda, \lambda / 2}\left(\Sigma_{0}\right), \quad \psi_{m} \in \mathrm{H}^{\lambda, \lambda 2}\left(\Sigma_{m}\right), \quad m=1,2 .
\end{gathered}
$$

We will assume that for the function $f_{m}, \varphi_{m}, \psi_{m}, m=1,2, z, \theta$, from (8)-(13) the agreement conditions hold at $t=0$ and these conditions are determined by a given the boundary conditions (12), (13) and the conjugation condition (11).

Then the following statement is true.

Theorem. Let for the coefficients of the operators $L_{m}, m=1,2$, and $L_{4}, L_{5}, L_{6}$ conditions (A1), (A2) and (B1), (B2) hold, respectively, for the surfaces $\Sigma_{m}$, $m=0,1,2$, and the functions $f_{m}, \varphi_{m}, \psi_{m}, m=1,2, z, \theta$ from the right-hand side (8)-(13) the conditions (14) and (15) hold. Then the problem (8)-(13) has a unique solution 


$$
u_{m} \in \mathrm{H}^{2+\lambda,(2+\lambda) / 2}\left(\bar{\Omega}_{m}\right), \quad m=1,2,
$$

in the performance the appropriate agreement conditions and the estimation

$$
\begin{aligned}
& \sum_{s=1}^{2}\left\|u_{m}\right\|_{\mathrm{H}^{2+\lambda,(2+\lambda) / 2}\left(\bar{\Omega}_{m}\right)} \leq C\left[\sum_{m=1}^{2}\left\|f_{m}\right\|_{\mathrm{H}^{\lambda, \lambda / 2}\left(\bar{R}_{T}^{n+1}\right)}+\sum_{m=1}^{2}\left\|\varphi_{m}\right\|_{\mathrm{H}^{2+\lambda}\left(R^{n}\right)}+\right. \\
& \left.+\|z\|_{\mathrm{H}^{2+\lambda,(2+\lambda) / 2\left(\Sigma_{0}\right)}}+\|\theta\|_{\mathrm{H}^{\lambda, \lambda / 2\left(\Sigma_{0}\right)}}+\sum_{m=1}^{2}\left\|\psi_{m}\right\|_{\mathrm{H}^{\lambda, \lambda / 2}\left(\Sigma_{m}\right)}\right]
\end{aligned}
$$

is true.

Proof. We will look for the solution of the problem (8)-(13) in the form

$$
u_{m}(x, t)=\sum_{s=0}^{3} u_{m}^{(s)}(x, t), \quad(x, t) \in \Omega_{m}, \quad m=1,2,
$$

of the sum of the simple-layer potentials (2), (3) with the unknown densities $V_{m}(\xi, \tau), V_{m+2}(\xi, \tau)$ and the potentials (4), (5) with the known functions $f_{m}(\xi, \tau)$, $\varphi_{m}(\xi)$. Using the properties of these potentials, we will find the unknown functions $V_{m}$ and $V_{m+2}, m=1,2$, so that for $u_{m}, m=1,2$, the conditions (10)-(13) have been met.

Let us consider a priori that unknown densities $V_{m}$ and $V_{m+2}, m=1,2$, satisfy the conditions

$$
V_{m} \in \underset{0}{\mathrm{H}^{\lambda, \lambda / 2}}\left(\Sigma_{0}\right), \quad V_{m+2} \in \underset{0}{\mathrm{H}^{\lambda, \lambda / 2}}\left(\Sigma_{m}\right), \quad m=1,2 .
$$

Now we pass to investigating the conjugation condition by Wentzel type (11). First, we transform this equality by separating its tangential and conormal components in the expressions that contain the derivatives of the first order in space variables using ratio

$$
\left(\boldsymbol{\beta}^{(0 m)}(x, t), \nabla u_{m}\right)=\sum_{i=1}^{n} \beta_{i}^{(0 m)}(x, t) \widetilde{\delta}_{i}^{(0 m)} u_{m}+\gamma_{0 m}(x, t) \frac{\partial u_{m}(x, t)}{\partial \mathbf{N}^{(0 m)}(x, t)}, \quad m=1,2,
$$

where $\widetilde{\delta}_{i}^{(0 m)}=D_{i}-\frac{v_{i}^{(0)}}{\left(\mathbf{N}^{(0 m)}, \mathbf{v}^{(0)}\right)} \sum_{k=1}^{n} N_{k}^{(0 m)} D_{k}, i=1, \ldots, n, m=1,2$, is a tangent differential operator on $\Sigma_{0} \backslash S_{0}, \gamma_{0 m}(x, t)=\frac{\left(\boldsymbol{\beta}^{(0 m)}(x, t), \mathbf{v}^{(0)}(x, t)\right)}{\left(\mathbf{N}^{(0 m)}(x, t), \mathbf{v}^{(0)}(x, t)\right)}, m=1,2$.

Then using (20) and the relationship from the theorem on the jump of the conormal derivative from the simple-layer potential (see [4]), we can write the condition (11) as 


$$
\begin{gathered}
\widetilde{L}_{4} u_{1}(x, t) \equiv \sum_{i, j=1}^{n} \beta_{i j}^{(0)}(x, t) \delta_{i}^{(0)} \delta_{j}^{(0)} u_{1}-\sum_{i=1}^{n} \beta_{i}^{(01)}(x, t) \widetilde{\delta}_{i}^{(01)} u_{1}+\sum_{i=1}^{n} \beta_{i}^{(02)}(x, t) \widetilde{\delta}_{i}^{(02)} u_{1}+ \\
+\left(\beta_{0}^{(01)}(x, t)+\beta_{0}^{(02)}(x, t)\right) u_{1}-D_{t} u_{1}=\theta^{(0)}(x, t), \quad(x, t) \in \Sigma_{0} \backslash S_{0},
\end{gathered}
$$

where

$$
\begin{gathered}
\theta^{(0)}(x, t)=\tilde{\theta}(x, t)+\sum_{m=1}^{2}(-1)^{m-1} \gamma_{0 m}(x, t) \frac{\partial u_{m}(x, t)}{\partial \mathbf{N}^{(0 m)}(x, t)}, \\
\tilde{\theta}(x, t)=\theta(x, t)+\beta_{0}^{(02)}(x, t) z(x, t)+\sum_{i=1}^{n} \beta_{i}^{(02)}(x, t) \widetilde{\delta}_{i}^{(02)} z(x, t), \\
\frac{\partial u_{m}(x, t)}{\partial \mathbf{N}^{(0 m)}(x, t)}=(-1)^{m-1} \frac{1}{2} V_{m}(x, t)+\int_{0}^{t} d \tau \int_{S_{\tau}^{(0)}} \frac{\partial G^{(m)}(x, t ; \xi, \tau)}{\partial \mathbf{N}^{(0 m)}(x, t)} V_{m}(\xi, \tau) d s_{\xi}+ \\
+\int_{0}^{t} d \tau \int_{S_{\tau}^{(m)}} \frac{\partial G^{(m)}(x, t ; \xi, \tau)}{\partial \mathbf{N}^{(0 m)}(x, t)} V_{m+2}(\xi, \tau) d s_{\xi}+\sum_{l=2}^{3} \frac{\partial u_{m}^{(l)}(x, t)}{\partial \mathbf{N}^{(0 m)}(x, t)}, \quad m=1,2 .
\end{gathered}
$$

For the kernel $\frac{\partial G^{(m)}(x, t ; \xi, \tau)}{\partial \mathbf{N}^{(0 m)}(x, t)}, m=1,2$, in the first and second integral from (22) the estimations $\left((x, t) \in \Sigma_{0} \backslash S_{0},(\xi, \tau) \in\left(\Sigma_{0} \cup \Sigma_{m}\right) \cap\{0 \leq \tau<t \leq T\}\right)$

$$
\left|\frac{\partial G^{(m)}(x, t ; \xi, \tau)}{\partial \mathbf{N}^{(0 m)}(x, t)}\right| \leq C(t-\tau)^{-\frac{n+1-\lambda}{2}} \exp \left\{-c \frac{|x-\xi|^{2}}{t-\tau}\right\}, \quad m=1,2
$$

are true.

Now, consider (21) as the autonomic parabolic equation on $\Sigma_{0} \backslash S_{0}$ for the function $u_{1}$. For its solution we introduce the following transformation of the variables:

$$
(x, t) \rightarrow(\widetilde{x}, \tilde{t}), \quad \tilde{x}_{i}=x_{i}, \quad i=1, \ldots, n-1, \quad \tilde{x}_{n}=x_{n}-F_{0}\left(x^{\prime}, t\right), \quad \tilde{t}=t .
$$

The conjugation condition (21) in new variables will take the form:

$$
\begin{array}{r}
\overline{\widetilde{L}}_{4} \bar{u}_{1} \equiv \sum_{k, l=1}^{n-1} \overline{\widetilde{\beta}}_{k l}^{(0)}\left(\widetilde{x}^{\prime}, \widetilde{t}\right) D_{k} D_{l} \bar{u}_{1}+\sum_{k=1}^{n-1} \overline{\widetilde{\beta}}_{k}^{(0)}\left(\widetilde{x}^{\prime}, \widetilde{t}\right) D_{k} \bar{u}_{1}+\overline{\widetilde{\beta}}_{0}^{(0)}\left(\widetilde{x}^{\prime}, \widetilde{t}\right) \bar{u}_{1}- \\
-D_{\tilde{t}} \bar{u}_{1}=\bar{\theta}^{(0)}\left(\widetilde{x}^{\prime}, \widetilde{t}\right), \quad\left(\widetilde{x}^{\prime}, \widetilde{t}\right) \in R_{T}^{n},
\end{array}
$$

where 


$$
\begin{gathered}
\overline{\widetilde{\beta}}_{k l}^{(0)}=\sum_{i, j=1}^{n} \bar{\beta}_{i j}^{(0)} \bar{\tau}_{i k}^{(0)} \bar{\tau}_{j l}^{(0)}, \quad k, l=1, \ldots, n-1, \quad \overline{\widetilde{\beta}}_{0}^{(0)}\left(\widetilde{x}^{\prime}, \widetilde{t}\right)=\bar{\beta}_{0}^{(01)}+\bar{\beta}_{0}^{(02)}, \\
\overline{\widetilde{\beta}}_{k}^{(0)}=\bar{\beta}_{k}^{(02)}-\bar{\beta}_{k}^{(01)}+\overline{\widetilde{\gamma}}_{01} \overline{\widetilde{a}}_{k n}^{(1)}-\overline{\widetilde{\gamma}}_{02} \overline{\widetilde{\alpha}}_{k n}^{(2)}-\sum_{i, j=1}^{n} \bar{\beta}_{i j}^{(0)} \delta_{i}^{(0)}\left(\bar{v}_{j}^{(0)} \cdot \bar{v}_{k}^{(0)}\right), \quad k=1, \ldots, n-1, \\
\overline{\widetilde{\gamma}}_{01}=\left(\overline{\widetilde{a}}_{n n}^{(1)}\right)^{-1}\left(\bar{\beta}_{n}^{(01)}-\sum_{i=1}^{n-1} \bar{\beta}_{i}^{(01)} D_{i} F_{0}-D_{t} F_{0}\right), \quad \overline{\widetilde{\gamma}}_{02}=\left(\overline{\widetilde{a}}_{n n}^{(2)}\right)^{-1}\left(\bar{\beta}_{n}^{(02)}-\sum_{i=1}^{n-1} \bar{\beta}_{i}^{(02)} D_{i} F_{0}\right), \\
\bar{\theta}^{(0)}=\overline{\widetilde{\theta}}+\sum_{m=1}^{2}(-1)^{m-1} \overline{\widetilde{\gamma}}_{0 m} \frac{\partial \bar{u}_{m}}{\partial \widetilde{\widetilde{N}}^{(0 m)}}, \quad \overline{\widetilde{\theta}}=\bar{\theta}+\bar{\beta}_{0}^{(02)} \bar{z}+\sum_{i=1}^{n-1}\left(\bar{\beta}_{i}^{(02)}-\overline{\widetilde{\gamma}}_{02} \overline{\widetilde{\sigma}}_{k n}^{(2)}\right) D_{k} \bar{z} .
\end{gathered}
$$

If follows from the conditions of Theorem, the additional assumption (19), formulae (25) and properties of the potentials that the coefficients and the function on the right-hand side of this equation belong to the space $\mathrm{H}^{\lambda, \lambda / 2}\left(\bar{R}_{T}^{n}\right)$. It is known that, the unique solution $u_{1}$ of the equation (24) which satisfies the initial condition $\bar{u}_{1}\left(\widetilde{x}^{\prime}, 0\right)=\bar{\varphi}_{1}\left(\widetilde{x}^{\prime}\right), \tilde{x}^{\prime} \in R^{n-1}$, can be represented by the formula

$$
\bar{u}_{1}\left(\widetilde{x}^{\prime}, \widetilde{t}\right)=\int_{R^{n-1}} \bar{P}\left(\tilde{x}^{\prime}, \widetilde{t} ; \widetilde{\xi}^{\prime}, 0\right) \bar{\varphi}_{1}\left(\widetilde{\xi}^{\prime}\right) d \widetilde{\xi}^{\prime}-\int_{0}^{t} d \tau \int_{R^{n-1}} \bar{P}\left(\tilde{x}^{\prime}, \tilde{t} ; \tilde{\xi}^{\prime}, \widetilde{\tau}\right) \bar{\theta}^{(0)}\left(\tilde{\xi}^{\prime}, \tilde{\tau}\right) d \tilde{\xi}^{\prime},
$$

where $\bar{P}\left(\widetilde{x}^{\prime}, \tilde{t}^{\prime} \widetilde{\xi}^{\prime}, \widetilde{\tau}\right)\left(\widetilde{x}^{\prime}, \widetilde{\xi}^{\prime} \in R^{n-1}, 0 \leq \widetilde{\tau}<\tilde{t} \leq T\right)$ is a f.s. of the uniformly parabolic equation $\widetilde{\widetilde{L}}_{4} \bar{u}_{1}=0$. Returning to the variables $(x, t)$ we can write equality (26) as

$$
u_{1}(x, t)=\int_{S_{0}} \Gamma(x, t ; \xi, 0) \varphi_{1}(\xi) d s_{\xi}-\int_{0}^{t} d \tau \int_{S_{\tau}^{(0)}} \Gamma(x, t ; \xi, \tau) \theta^{(0)}(\xi, \tau) d s_{\xi}, \quad(x, t) \in \Sigma_{0},
$$

where $\Gamma(x, t ; \xi, \tau)=P(x, t ; \xi, \tau) \cdot v_{n}^{(0)}(\xi, \tau), \quad(x, t) \in \Sigma_{0}, \quad(\xi, \tau) \in \Sigma_{0}, \quad 0 \leq \tau<t \leq T$. The function $u_{1}(x, t),(x, t) \in \Sigma_{0}$ belongs to class $\mathrm{H}^{2+\lambda,(2+\lambda) / 2}\left(\Sigma_{0}\right)$ as well.

Thus, we have two representations for values of function $u_{1}$ on $\Sigma_{0}$ : relation (18), where one should put $m=1,(x, t) \in \Sigma_{0}$ and relation (27). Then, comparing the right-hand sides of equalities (18) and (27) and taking into account (25), we obtain the first integral equation for the unknown functions $V_{m}$ and $V_{m+2}, m=1,2$. Using the equality (27) and the conjugation condition (10), we find the second equation for these functions. The third and fourth equations of the required system for $V_{m}$ and $V_{m+2}, m=1,2$, we obtain from the boundary conditions (12) and (13) similar to the way in which we found the first equation. After appropriate transformations, an obtained system of four equations for the unknown functions $V_{m}$ and $V_{m+2}, m=1,2$, can be represented as 


$$
\begin{aligned}
& \int_{0}^{t} d \tau \int_{S_{\tau}^{(0)}} G^{(m)}(x, t ; \xi, \tau) V_{m}(\xi, \tau) d s_{\xi}+\sum_{l=1}^{2} \int_{0}^{t} d \tau \int_{S_{\tau}^{(0)}} K_{m l}(x, t ; \xi, \tau) V_{l}(\xi, \tau) d s_{\xi}+ \\
& +\sum_{l=1}^{2} \int_{0}^{t} d \tau \int_{S_{\tau}^{(l)}} K_{m, l+2}(x, t ; \xi, \tau) V_{l+2}(\xi, \tau) d s_{\xi}=\Phi_{m}(x, t), \quad(x, t) \in \Sigma_{0}, m=1,2, \\
& \int_{0}^{t} d \tau \int_{S_{\tau}^{(m)}} G^{(m)}(x, t ; \xi, \tau) V_{m+2}(\xi, \tau) d s_{\xi}+\int_{0}^{t} d \tau \int_{S_{\tau}^{(0)}} K_{m+2, m}(x, t ; \xi, \tau) V_{m}(\xi, \tau) d s_{\xi}+ \\
& +\int_{0}^{t} d \tau \int_{S_{\tau}^{(m)}} K_{m+2, m+2}(x, t ; \xi, \tau) V_{m+2}(\xi, \tau) d s_{\xi}=\Phi_{m+2}(x, t), \quad(x, t) \in \Sigma_{m}, m=1,2,
\end{aligned}
$$

where

$$
\begin{gathered}
\Phi_{m}(x, t)=\Phi_{0}(x, t)-\sum_{l=2}^{3} u_{m}^{(l)}(x, t)-(m-1) z(x, t), \quad m=1,2, \\
\Phi_{0}(x, t)=-\int_{0}^{t} d \tau \int_{S_{\tau}^{(0)}} \Gamma(x, t ; \xi, \tau)\left[\tilde{\theta}(\xi, \tau)+\sum_{k=1}^{2} \sum_{l=2}^{3}(-1)^{k-1} \gamma_{0 k}(\xi, \tau) \frac{\partial u_{k}^{(l)}(\xi, \tau)}{\partial \mathbf{N}^{(0 k)}(\xi, \tau)}\right] d s_{\xi}+ \\
+\int_{S_{0}} \Gamma(x, t ; \xi, 0) \varphi_{1}(\xi) d s_{\xi}, \\
\Phi_{m+2}(x, t)=-\int_{0}^{t} d \tau \int_{S_{\tau}^{(m)}} \Gamma_{m}(x, t ; \xi, \tau)\left[\psi_{m}(\xi, \tau)+\gamma_{m}(\xi, \tau) \sum_{l=2}^{3} \frac{\partial u_{m}^{(l)}(\xi, \tau)}{\partial N^{(m)}(\xi, \tau)}\right] d s_{\xi}+ \\
+\int_{S_{m}} \Gamma_{m}(x, t ; \xi, 0) \varphi_{m}(\xi) d s_{\xi}-\sum_{l=2}^{3} u_{m}^{(l)}(x, t), \quad(x, t) \in \Sigma_{m}, m=1,2, \\
\gamma_{m}(x, t)=\frac{\left(\boldsymbol{\beta}^{(m)}(x, t), \mathbf{v}^{(m)}(x, t)\right)}{\left(\mathbf{N}^{(m)}(x, t), \mathbf{v}^{(m)}(x, t)\right)}, \quad(x, t) \in \Sigma_{m}, m=1,2 .
\end{gathered}
$$

$\Gamma_{1}$ and $\Gamma_{2}$ are the f.s. of the parabolic equations which we obtained after transformation of the boundary conditions (12) and (13) using the scheme to obtain the equation (21). The kernels $K_{m l}, K_{m, l+2}, K_{m+2, m}, K_{m+2, m+2}, m=1,2, l=1,2$, are expressed by the functions which have a „weaker" singularity than the function $G^{(m)}(x, t ; \xi, \tau)$ at $\tau \uparrow t$.

So, we have a system of four integral Volterra equations of the first kind (28) for $V_{m}$ and $V_{m+2}, m=1,2$. The functions $\Phi_{m}(x, t), \quad(x, t) \in \Sigma_{0}, m=1,2$, and $\Phi_{m+2}(x, t),(x, t) \in \Sigma_{m}, m=1,2$, from right-hand side of equations of this system 
belong to the Holder classes $\underset{0}{H^{2+\lambda,(2+\lambda) / 2}}\left(\Sigma_{0}\right)$ and $\underset{0}{H^{2+\lambda,(2+\lambda) / 2}}\left(\Sigma_{m}\right)$ respectively. In order to transform each of the equations of this system, we introduce the special integro-differential operators similar to the operator that was introduced (see $[4,8$, 13-16]) in the study of the first boundary-value parabolic problem by the boundary integral equation method.

Let $(x, t) \in \Sigma_{0} \backslash S_{0}$. In this case, the integro-differential operators (denote them by $\mathcal{E}_{m}, m=1,2$ ) which will be used to transform the first two equations of system (28), can be defined by the formula

$$
\mathcal{E}_{m}(x, t) \psi=\left.\frac{2}{\sqrt{\pi}}\left\{\frac{\partial}{\partial t} \int_{0}^{t}(t-\tau)^{-1 / 2} d \tau \int_{S_{\tau}^{(0)}} H^{(0 m)}(x, \hat{t} ; \xi, \tau) \psi(\xi, \tau) d s_{\xi}\right\}\right|_{\hat{t}=t} .
$$

Here the function $H^{(0 m)}(x, \hat{t} ; \xi, \tau),\left((x, t) \in \Sigma_{0} \backslash S_{0},(\xi, \tau) \in \Sigma_{0}, 0 \leq \tau<t \leq T\right)$, $m=1,2$, is a f.s. of the uniformly parabolic operator, which is a trace of the operator $L_{m}, m=1,2$, on $\Sigma_{0}$. To transform the third and fourth equations of the system (28), we use the integro-differential operators $\mathcal{E}_{m+2}, m=1,2$, which are similar to the operator $E_{m}, m=1,2$, from (29). To this end on the right-hand side (29) we should replace the function $H^{(0 m)}(x, t ; \xi, \tau)$ and integrate over the surface $S_{\tau}^{(0)}$ to the function $H^{(m)}(x, t ; \xi, \tau)$ and integrate over the surface $S_{\tau}^{(m)}$ respectively. Here $H^{(m)}(x, t ; \xi, \tau) \quad\left((x, t) \in \Sigma_{m} \backslash S_{m},(\xi, \tau) \in \Sigma_{m}, 0 \leq \tau<t \leq T\right)$ is a f.s. of the parabolic operator, which is a trace of the operator $L_{m}$ on $\Sigma_{m}, m=1,2$.

Applying $\mathcal{E}_{m}$ and $\mathcal{E}_{m+2}, m=1,2$, to both sides of the corresponding equations of the system (28), we transform this system into the equivalent system of the integral Volterra equations of the second kind

$$
\begin{gathered}
V_{m}(x, t)+\sum_{l=1}^{2} \int_{0}^{t} d \tau \int_{S_{\tau}^{(0)}} R_{m l}(x, t ; \xi, \tau) V_{l}(\xi, \tau) d s_{\xi}+ \\
+\sum_{l=1}^{2} \int_{0}^{t} d \tau \int_{S_{\tau}^{(l)}} R_{m, l+2}(x, t ; \xi, \tau) V_{l+2}(\xi, \tau) d s_{\xi}=\Psi^{(m)}(x, t), \quad(x, t) \in \Sigma_{0} \backslash S_{0}, \quad m=1,2 \\
V_{m+2}(x, t)+\int_{0}^{t} d \tau \int_{S_{\tau}^{(0)}} R_{m+2, m}(x, t ; \xi, \tau) V_{m}(\xi, \tau) d s_{\xi}+ \\
+\int_{0}^{t} d \tau \int_{S_{\tau}^{(m)}} R_{m+2, m+2}(x, t ; \xi, \tau) V_{m+2}(\xi, \tau) d s_{\xi}=\Psi^{(m+2)}(x, t), \quad(x, t) \in \Sigma_{m} \backslash S_{m}, \quad m=1,2,
\end{gathered}
$$


where

$$
\begin{gathered}
\Psi^{(m)}(x, t)=\left(\mathbf{A}_{m}(x, t) \mathbf{v}^{(0)}(x, t), \mathbf{v}^{(0)}(x, t)\right)^{1 / 2} \mathcal{E}_{m}(x, t) \Phi_{m}, m=1,2, \\
\Psi^{(m+2)}(x, t)=\left(\mathbf{A}_{m}(x, t) \mathbf{v}^{(m)}(x, t), \mathbf{v}^{(m)}(x, t)\right)^{1 / 2} \mathcal{E}_{m+2}(x, t) \Phi_{m+2}, \quad m=1,2 .
\end{gathered}
$$

And for the kernels $R_{m l}, R_{m, l+2}, R_{m+2, m}, R_{m+2, m+2}, m=1,2, l=1,2$, the inequality (23) is true.

Solving the system of equations (30) by the method of subsequent approximations, we find $V_{m}, m=1,2,3,4$. One can additionally verify that $V_{m}$ and $V_{m+2}$, $m=1,2$, satisfy the condition (19).

We obtained the solution of the problem (8)-(13) by formulas (18), (30). To complete the proof of Theorem, we have to only check that this solution satisfies the condition (16) and the estimate (17). We have to also verify the statement of the Theorem on the uniqueness of this solution.

In this regard, we note that the strict proof of these facts practically repeats the similar statements in the papers [13-16]. The theorem is proved.

\section{Conclusions}

In the article, we investigated the question of the classic solvability of the parabolic initial-boundary problem with the boundary conditions and one Wentzel conjugation condition in the assumption that the boundaries of the domains are the elementary noncylindrical surfaces of Holder class $\mathrm{H}^{2+\lambda,(2+\lambda) / 2}$. The solution is obtained by the usual parabolic simple-layer potentials by using the boundary integral equation method. The proposed approach can be used to solve a similar conjugation problem in the noncylindrical domain of the more general type.

\section{References}

[1] Pogorzelski W., Etude de la solution fondamentale de l'équation parabolique, Richerce Matem. 1956, 5, 25-57.

[2] Friedman A., Partial Differential Equations of Parabolic Type, Prentice-Hall, Englewood Cliffs $1964,7$.

[3] Ladyzhenskaya O.A., Solonnikov V.A., Ural'tseva N.N., Linear and quasilinear equations for parabolic type, Nauka, Moskow 1967.

[4] Baderko E.A., Boundary problems for the parabolic equation and boundary integral equations, Differ. Equations. 1992, 28, 1, 17-23.

[5] Kamynin I.I., Application of Pagni parabolic potentials to boundary problems of mathematical physics, I, Differ. Equations 1990, 26, 5, 829-841.

[6] Ivasyshen S.D., Green Matrices of Parabolic Boundary Problem, Vyshcha shkola, Kiev 1990.

[7] Gytarashu N.V., Eydel'man S.D., Parabolic boundary problems, Kyshynev, Shtynitsa 1992. 
[8] Cherepova M.F., Solving of the first boundary problem for parabolic equation of the second order in noncylibdrical domain by the potential method, M., Dep. in VINITI 11.01.85, 361-385 Dep.

[9] Dynkin E.B., Markov Processes, Fizmatgiz, Moskow 1963.

[10] Wentzel A.D., On boundary conditions for multidimensional diffusion processes, Probab. Theory Appl. 1959, 4, 2, 172-185.

[11] Apushkinskaya D.E., Nazarov A.I., Initially-boundary problem with limit Ventzel condition for nondivergent parabolic equations, RAN, Algebra and Analysis 1994, 6, 6, 1-29.

[12] Yi Zeng, Yousong Luo, Linear Parabolic Equations with Ventsel Initial Boundary Conditions, Bull. Austral. Math. Soc. 1995, 51, 465-479.

[13] Kopytko B., Tsapovska Zh., The potential method in a parabolic boundary problem with boundary Wentsel condition, Visnyk Lviv Univ., Ser. Mech.-Math. 2000, 56, 106-115.

[14] Kopytko B.I., Tsapovska Zh.Ya., Integral Representation of an Operator Semigroup Describing a Diffusion in a Domain with Wentzel's Boundary Condition, Theory of Stochastic Processes 1999, 5(21), 3-4, 105-112.

[15] Kopytko B.I., Mylyo O.Ya., Tsapovska Zh.Ya., A parabolic conjugation problem with general boundary condition and conjugation condition of Wentzel type, Carpatian Mathematical Publications. 2010, 2, 2, 55-73.

[16] Kopytko B.I., Tsapovska Zh.Ya.. Initial boundary-value problem with Wentzel-type conjugation condition for a parabolic equation with discontinuous coefficients, Journal of Mathematical Sciences 2009, 160, 3, 283-295. 